\title{
Scale ratio ICP for 3D registration of coronary venous anatomy with left ventricular epicardial surface to guide CRT lead placement (Erratum)
}

, "Scale ratio ICP for 3D registration of coronary venous anatomy with left ventricular epicardial surface to guide CRT lead placement (Erratum)," Proc. SPIE 10951, Medical Imaging 2019: Image-Guided Procedures, Robotic Interventions, and Modeling, 109513B (14 June 2019); doi: $10.1117 / 12.2540856$

SPIE. Event: SPIE Medical Imaging, 2019, San Diego, California, United States 


\title{
Scale ratio ICP for 3D registration of coronary venous anatomy with left ventricular epicardial surface to guide CRT lead placement (Erratum)
}

\author{
Haipeng Tanga, Robert Boberb, Chaoyang Zhanga, Zhuo Hea, Jiangang Zouc, and Weihua Zhoua \\ aThe Univ. of Southern Mississippi (United States) \\ bOchsner Medical Ctr. (United States) \\ cFirst Affiliated Hospital of Nanjing Medical Univ. (China)
}

Proceedings Volume 10951, Medical Imaging 2019: Image-Guided Procedures, Robotic Interventions, and Modeling; 1095123 (2019) https://doi.org/10.1117/12.2512775

Event: SPIE Medical Imaging, 2019, San Diego, California, United States

Online Publication Date: 8 March 2019

Erratum Published: 14 June 2019

A revised version of this manuscript was published on 14 June 2019. Details of the revision are provided below. The original paper has been updated.

1, in Eq.2, a transpose symbol is added to the second term of the equation. Before $2 \sum_{i=1}^{N p} S \cdot R \tilde{p}_{i} \tilde{m}_{i}^{k}$, after $2 \sum_{i=1}^{N p} S \cdot\left(R \tilde{p}_{i}\right)^{(T)} \widetilde{m}_{i}^{k}$.

2, in Eq.3, a minus symbol is added to the matrix. Before $\left(\begin{array}{lll}1 & 0 & 0 \\ 0 & 1 & 0 \\ 0 & 0 & 1\end{array}\right)$, after $\left(\begin{array}{ccc}1 & 0 & 0 \\ 0 & 1 & 0 \\ 0 & 0 & -1\end{array}\right)$.

3, in Eq.4, a transpose symbol is added to the numerator. Before $\sum_{i=1}^{N p} R^{k+1} \tilde{p}_{i} \tilde{m}_{i}^{k}$, after $\sum_{i=1}^{N p}\left(R^{k+1} \tilde{p}_{i}\right)^{(\tau)} \tilde{m}_{i}^{k}$.

Medical Imaging 2019: Image-Guided Procedures, Robotic Interventions, and Modeling edited by Baowei Fei, Cristian A. Linte, Proc. of SPIE Vol. 10951, 109513B - (C) 2019 SPIE CCC code: $1605-7422 / 19 / \$ 18 \cdot$ doi: $10.1117 / 12.2540856$ 